\title{
A New Type of Steady and Stable, Laminar, Premixed Flame in Ultra-Lean, Hydrogen-Air Combustion
}

\author{
Joseph F. Grcar ${ }^{\mathrm{a}}$ \\ ${ }^{a}$ Center for Computational Science and Engineering \\ Lawrence Berkeley National Laboratory \\ Berkeley, CA 94720-8142, USA
}

\begin{abstract}
Ultra-lean, hydrogen-air mixtures are found to support another kind of laminar flame that is steady and stable beside flat flames and flame balls. Direct numerical simulations are performed of flames that develop into steadily and stably propagating cells. These cells were the original meaning of the word "flamelet" when they were observed in lean flammability studies conducted early in the development of combustion science. Several aspects of these two-dimensional flame cells are identified and are contrasted with the properties of one-dimensional flame balls and flat flames. Although lean hydrogen-air flames are subject to thermo-diffusive effects, in this case the result is to stabilize the flame rather than to render it unstable. The flame cells may be useful as basic components of engineering models for premixed combustion when the other types of idealized flames are inapplicable.
\end{abstract}




\section{Nomenclature}

$\phi$ fuel equivalence ratio

\section{Introduction}

There is growing evidence that a new type of laminar, premixed flame occurs in ultra-lean, hydrogen-air combustion. Recently, Kagan, Minaev, and Sivashinsky [1] hypothesized "an intermediate combustion mode realized through localized yet self-drifting ball-like flamelets." Earlier, Wu, Ronney, Colantonio, and VanZandt [2] found that "stable flame ball solutions and planar flame solutions do not coexist for any value of $\phi$," and for a short range of $\phi$, there are no known stable laminar flames of any kind.

This paper presents direct numerical simulations — with detailed chemistry and convection — to indicate that the hypothesized combustion mode exists and bridges the gap between the flat and ball flames. The flame structure appears to resemble that seen by Markstein and others in early observations of thermo-diffusively unstable flames (see $[3,4,5]$ and references therein).

\section{Conditions for the Known Basic Flames}

Flat flames and flame balls are two types of laminar, premixed flames. They are steady and stable in the following sense. The flames are modelled by time-dependent, one-dimensional (1D) differential equations. Integrating the equations in time evolves the solutions to steady-states. When the time derivatives approach zero, the flat flame propagates at a constant velocity which is an eigenvalue of the equations with zero time derivatives; similarly, the flame ball becomes motionless at a constant radius which is an eigenvalue of its equations. These steady solutions are stable in that, if slightly perturbed, they can be recovered by integrating further in time.

There is reason to believe there should be another basic type of laminar, premixed flame. One piece of evidence is the lack of flat flames or flame balls for some hydrogen-air mixtures. Buckmaster, Smooke, and Giovangigli 
[6] numerically confirmed the theoretical predictions of Buckmaster, Joulin, and Ronney [7, 8] that steady flame balls can exist only between certain lean and rich limits. Wu, Ronney, Colantonio, and VanZandt [2] discovered the solutions are unstable when $0.285<\phi$. They also found the flat-flame equations, with radiative losses, do not have stable solutions when $\phi<0.296$. Wu et al. concluded, if $\phi$ is chosen in the overlap region of these two conditions, namely if $0.285<\phi<0.296$, then there are no known, steady and stable, laminar, hydrogen-air flames for this value of $\phi$. The specific limits may depend on the choice of reaction mechanism and transport properties, but they must be regarded as approximately correct in view of the careful theoretical and numerical analysis supporting them.

Additional evidence for another basic type of laminar, premixed flame are observations of flames that propagate through mixtures that are too lean to support flat flames. These observations are associated with the determination of flammability limits such as by the United States Bureau of Mines $[9,10]$, which considered the propagation of flames in vertical tubes. The lean flammability limits for hydrogen-air mixtures determined in this way are about $\phi=0.1$ (4\% volume of fuel) for upwardly propagating flames [9], and $\phi=0.23$ (9\%) for downwardly propagating flames [3, p. 10]. Markstein performed his own experiments of this kind, in papers summarized in [3], and he surveyed earlier work, noting that Coward and Brinsley appear to have been the first to report "a rising ball of flame, which frequently broke up into a number of small rising flamelets" [3, p. 10]. The books of both Markstein and of Lewis and von Elbe gave particular credence to the observations of Clusius and coworkers; some hand sketches are reproduced in [4, p. 315]. Similar observations with photographs were later reported in the two cited books, and by Bregeon, Gordon, and Williams [11], and by Mitani and Williams [12].

Hertzberg [5, p. 223] summarized all the descriptions of the leanest hydrogen flames as follows: "the cells are so widely dispersed that their boundaries do not touch one another, and the dark, quenched zones occupy a large fraction of the tube cross section." On one hand, the observed flames cannot be regarded as perturbations of flat flames, because the flat flame equations with radiative losses do not have solutions for such lean hydrogen-air mixtures. On the other hand, the observed flames cannot be flame balls which are necessarily motionless, in theory, due to spherical symmetry. Flame balls can drift in the direction of mild thermal and fuel gradients, but the motion 
is very slow, $\mathcal{O}(1) \mathrm{cm} / \mathrm{min}$ having been observed in NASA microgravity experiments that included a hydrogen-air mixture with $\phi=0.088$ [13]. In short, neither flat flames nor flame balls can be found in the flames that determine the lean flammability limits of hydrogen-air mixtures.

\section{Computational Experiments}

\subsection{Rationale}

If another type of laminar flame is to be steady and stable, then evidently it must be nonplanar and aspherical, lest it become a flat flame or a flame ball. To find the hypothesized flame, the present work proceeds by analogy with flat flames and flame balls. Those flames are steady and stable in some idealized circumstances, so other types of laminar flames may be steady and stable under different circumstances. Thus, the experiment consists of solving the reacting flow equations in two-dimensional (2D) domains with periodic boundaries beginning with an artificially wrinkled flame extending horizontally across the width of the domain, and with fresh gas below and burnt gas above (boundary conditions: inflow at the bottom, outflow at the top, and periodic at the left and right). The flame is then allowed to evolve to see whether a steady and stable flame develops, and if so, what kind.

\subsection{Equations and Models}

The calculations are performed by the software and algorithms described by Day and Bell [14]. The equations for viscous, reacting flow of multicomponent, ideal gases are so well known they need not be stated here, but if necessary they and the algorithm used to solve them can be found in the previously mentioned publication, [14], and in the references therein, such as $[15,16,17,18,19]$. Calculations made with this methodology have compared well with turbulent flame experiments [20,21].

The mixture-averaged model of diffusion is used with the properties computed by the EGLIB software of Ern and Giovangigli $[22,23]$. The chemical mechanism is the sub-mechanism of GRI-Mech 2.11 [24] consisting of $\mathrm{N}_{2}$ 
and of the species containing only $\mathrm{H}$ and $\mathrm{O}$ atoms. This mechanism has been deemed appropriate in studies of flame balls [25].

The spatial resolution of the calculations is 117 microns, which is more than adequate for the very thick reaction zones encountered. For example, at the leading edge of the flame cells to be investigated, the thermal thickness is 5 $\mathrm{mm}$ based on a linear fit to temperature at the point of steepest gradient. There the fuel consumption zone is $1 \mathrm{~mm}$ thick based on the full width at half maximum.

The choice of periodic boundary conditions is somewhat arbitrary. For the one-dimensional equations that describe flat flames and flame balls, only the boundary conditions for burnt and fresh mixtures are needed. For two-dimensional equations, clearly, some additional boundary conditions are required at the sides of the domain. Channels with adiabatic walls could be used to more closely match the conditions of the experiments, cited in section 2, for determining lean extinction limits. However, periodic boundaries are frequently used in computational studies of laminar flame instabilities as for example in many of the calculations surveyed in section 3.4, below. Periodic boundaries do add an additional degree of freedom by permitting lateral motion which is sometimes seen in calculations of flames exhibiting the Landau Darrieus instability.

\subsection{Experimental Cases}

The specifics of the experiments are these. As with flat flames and flame balls, gravity is neglected. Radiative losses are also neglected here, the significance of which is discussed below. The fresh and inflowing mixture is hydrogenair at $\phi=0.2$. To ensure that a flame ignites in such a lean mixture, the calculation begins from the flame zone and burnt gas of a richer mixture, $\phi=0.3$, as determined by the standard CHEMKIN software, [26]. The flame zone quickly adapts to the leaner mixture, and the initially richer exhaust completely flushes out during the calculation. The temperature of the fresh gas is $300 \mathrm{~K}$ and the ambient pressure is $1.0 \mathrm{~atm}$.

Ten domains of different widths are used, from 2.0 to $6.0 \mathrm{~cm}$ in steps of $0.5 \mathrm{~cm}$, and one domain of width $7.5 \mathrm{~cm}$. All domains have the same height, $9.0 \mathrm{~cm}$, except the widest has height $12.0 \mathrm{~cm}$. During the numerical experiments, 
the inflow of fresh gas at the bottom boundary is adjusted to maintain a constant volume of fresh mixture equal to one quarter the domain volume using the control method described in [27]. The model time duration for each experiment is $10.0 \mathrm{sec}$, after which the state of the flame is determined.

\subsection{Relation to Previous Numerical Experiments}

The present numerical experiments differ in intent and conditions from previous work. Studies of propagating flames have focused on either forced or intrinsic instabilities, and may or may not have exhibited cells that are separated by extinguished zones. Additionally, the prior direct numerical simulations of hydrogen-air flames with detailed chemical mechanisms have considered richer mixtures than those of interest here. In what may be the earliest such simulations, Patnaik, Kailasanath, Laskey, and Oran [28] verified that the thermo-diffusive instability causes flames to wrinkle in mixtures with $\phi \approx 0.3$. Patnaik and Kailasanath [29] found extinguished regions in later calculations. Tanahashi, Fujimura, and Miyauchi [30] examined vortex-flame interactions in turbulent mixtures with $\phi=1.0$. Fluctuating cells separated by narrow extinguished zones were observed by Bell, Cheng, Day, and Shepherd [31] in turbulent flames, and by Grcar, Bell, and Day [32] in laminar flames, in both cases with $\phi=0.37$.

The many computational studies of intrinsic flame instabilities generally have used simplified governing equations that do not allow a fuel equivalence ratio, $\phi$, to be associated with the mixture. The earliest such calculations based on the Kuramoto-Sivashinsky equation may have been those of Michelson and Sivashinsky [33]. They incorporate fluid properties through the Lewis number of the deficient reactant and through the ratio of densities in the fresh and burnt gases. Denet and Haldenwang [34, 35] studied instabilities with either reaction-diffusion equations or the Navier-Stokes equations, in both cases with a single reactant. The Navier-Stokes equations again with a single reactant were also used by Kadowaki in a series of papers summarized in [36]. In this formulation, for example in [37], the Navier-Stokes equations are nondimensionalized by the burning velocity of the adiabatic, planar flame for the given conditions. A scalloped flame characteristic of the Landau-Darrieus instability advances steadily for some Lewis numbers and planar burning velocities [37, p. 179, figs. 5, 6] but local extinction was not observed. Yuan, 
Ju, and Law [38] performed similar calculations and did find the extinguished regions between flame cells that are expected when the Lewis number of the deficient reactant is much less than one. Their interest was the unstable dynamics of many cells and thus they did not investigate the properties of individual cells nor did they exhibit stable cells that form closed loops (these being two dimensional calculations).

The relatively few calculations of isolated flame balls in lean mixtures have also used simplified governing equations. Gerlinger, Schneider, Föhlich, and Bockhorn [39] and Kagan, Minaev, and Sivashinsky [1, 40] solved reaction-diffusion equations. As in the studies of laminar flame instabilities, they employed nondimensionalization and one-step or idealized reaction source terms.

When nondimensionalized equations are used, the conclusions of the prior work apply in principle to any mixture characterized by the given values of the dimensionless quantities. The generality of nondimensionalization may be illusory, however, because the spatial and temporal scaling depends on properties of adiabatic, planar flames that may not be physically meaningful for ultra-lean mixtures. Such flames have been calculated by several groups such as by Giovangigli and Smooke [41]. However, the solutions obtained for ultra-lean mixtures are very thick, and further, Lozinski, Buckmaster, and Ronney [42] note an interaction between the solution of the flat flame equations and the boundary conditions. In this case it then becomes unclear how to interpret the nondimensionalization.

Finally, adiabatic flame balls are predicted to be always unstable [43, 44] so volumetric heat loss from thermal radiation is necessary for their existence [7]. The radiation stabilizes the ball by preventing the buildup of heat. Lacking some means of heat loss besides diffusion, the warm zone of the flame ball would expand continually as the low Lewis number fuel diffuses into the flame more rapidly than heat can diffuse away. Thus the inclusion of radiative heat loss was a prominent feature of the calculations of flame balls previously cited. It is plausible to neglect radiation here because the present hydrogen-air mixture is much richer than those in which NASA experiments found flame balls. Omitting radiation provides a test of whether there are other stabilizing mechanisms. 


\section{Results}

\subsection{Existence of Steady and Stable Flame Cells}

Steady and stable flame cells do form in the domains of widths from $3.0 \mathrm{~cm}$ to $4.5 \mathrm{~cm}$. The flame cells have the hemispherical shape with trailing tails described by Markstein in laboratory experiments, and in several more recent papers about reaction-diffusion systems by Brailovsky, Kagan, Minaev, and Sivashinsky [1, 40, 45]. The novelty of the present observations is that steady and stable flame cells do form in systems governed by the reacting flow equations, with detailed chemical mechanisms, and even without the stabilizing influence of radiative losses. Moreover, the thermo-diffusive instability of lean hydrogen-air flames is usually associated with unsteady and unstable behavior, whereas here it will be seen, the thermo-diffusive effect promotes the existence of a steady and stable flame.

Table 1 lists the outcome of each experiment. In the narrowest domains the initially wrinkled flames evolve to flat flames that would extinguish if the calculation included radiative losses. In two wider domains the flames evolve to flames that may be considered steady although they precess horizontally across the domain. This form of motion is allowed by the periodic boundaries at the left and right. A precessing flame approaches the incoming fluid at a nonzero angle of attack, so the domain has been effectively narrowed, suggesting steady flames do not exist for the full domains of these nominal widths. In the wider domain the flame exhibits a repetitive oscillating motion. In the very widest domain, two flame cells develop that slowly vary in size as each tries to outrace the other; this case pertains to the suggestion [46] that standardized flame cells can be used to build engineering models of ultra-lean flames. The remainder of this paper discusses the steady and stable cases.

To check the singularity of the findings in Tab. 1, some additional simulations were conducted for leaner and richer mixtures, $\phi=0.15$ and 0.30 , and for a few domain widths. The leaner mixtures extinguished, while the richer mixtures produced flames with narrow extinguished zones that can be seen in calculations and experiments in [31], and in calculations in [32]. Thus only for some range of mixtures including $\phi=0.2$ do there exist the 
widely dispersed flame cells described in the previously discussed surveys of Markstein [3], Lewis and von Elbe [4], and Hertzberg [5]. On an historical note, it is interesting to learn that the word "flamelet" originally meant precisely these dispersed flame cells (see, for example [3, pp. 10, 11]) rather than as the word is used today for a small piece of a stretched, planar flame in a wrinkled flame model. The vigorously burning flames that have very narrow extinguished zones originally were called "polyhedral" [5, p. 223].

\subsection{Flame Cell Sizes and Speeds of Propagation}

Like flame balls, the steady and stable flame cells are isolated flames. The width of each cell is roughly one-third to one-half the corresponding domain width, as shown in Fig. 1. It is not unexpected that a flame propagating in a domains with periodic boundaries should have a shape or size that depends on the width. For example, the one-cusp solution of the Kuramoto-Sivashinsky equation depends on the domain width [47]. At least for this two-dimensional model, both the cell width and the flame speed are linear functions of the domain width, with the smaller cells moving more quickly. Smaller cells in narrower domains move faster because there is greater magnification of diffusive flux of fuel to more sharply curved surfaces. Flames propagate toward fresh mixture, so in wider domains the flanks of cells move outward until diffusion can deplete the fuel further to the side. The large, more gently curved cells experience lower diffusive flux locally and therefore propagate more slowly. The flame cell widths are measured from crest to crest of fuel consumption at the widest extent. Since the inflow is adjusted to hold the flame cells steady in the frame of reference of the computational domain, the inflow velocity gives the propagation speed of the cell into a quiescent mixture. The flame cells move at $\mathcal{O}(1) \mathrm{cm} / \mathrm{sec}$, which is much faster than the $\mathcal{O}(1) \mathrm{cm} / \mathrm{min}$ observed for flame balls.

Since flame balls have not been reported to be capable of such propagation speeds, the laminar flame cells appear to be a more reasonable basis on which to build models of the cellular flames observed by Markstein and others. These results suggest that an ensemble of widely spaced flame cells could move unidirectionally, in unison, to form a propagating front [46]. That smaller cells are swifter indicates a compensation device whereby cells crowded out 
by encroaching neighbors could race ahead to reach the fresher mixture sooner. Indeed such has been observed in the $7.5 \mathrm{~cm}$ width calculation. Fig. 6 shows a pair of flame cells that persist side-by-side for $4.5 \mathrm{~s}$ until one falls behind and extinguishes; the survivor then divides and the process repeats.

The size and speed of flame cells reported in Fig. 1 are consistent with laboratory observations summarized by Hertzberg [5]. It is difficult to find experimental data that corresponds exactly to the very lean conditions studied here. For example, Mitani and Williams [12] consider $\mathrm{H}_{2}-\mathrm{O}_{2}-\mathrm{N}_{2}$ mixtures in which the lowest molecular hydrogenoxygen ratio is 0.96 , whereas the ratio is 0.4 in the present hydrogen-air flame with $\phi=0.2$. In their conditions which are both richer and include buoyancy, Mitani and Williams report that the cells have diameters of $1.2 \mathrm{~cm}$ or larger [12, p. 175, fig. 7], and some cells do propagate at speeds below $10 \mathrm{~cm} / \mathrm{s}$ [5, p. 224, fig. 7].

\subsection{Anisotropic Closed Boundaries around Cells}

A notable feature of the flame cells is that they are closed like the flame balls. As can be seen in Fig. 2, the local peak of fuel consumption forms a continuous boundary around an interior in which there is little or no fuel. This feature appears to be characteristic of the steady cells. Flames that did not become steady in wider domains did not form closed tails.

The flame cells generate a considerable temperature gradient as also shown in Fig. 2. This configuration differs from flame balls, whose medium can return to a uniform, ambient temperature within a distance of approximately one diameter from the flame ball. The cross section of temperature through the flame cell in Fig. 3 can be contrasted with that through a flame ball as predicted by Brailovsky and Sivashinsky [45, p. 527, fig. 3]. The temperature falls

off radially in both stationary and drifting the flame balls, whereas it remains elevated in the wake of the moving flame cell. The temperature also has a local minimum inside the flame balls, but not in the flame cells. These flame ball predictions include radiation while the present flame cell calculations do not, but this difference is somewhat ameliorated because hydrogen flames radiate significantly less heat than hydrocarbon flames. 


\subsection{Flame Cell Chemistry}

The thermo-diffusive instability is driven by the preferential diffusion of the reactant that is both deficient and has Lewis number less than 1 , which in this case is the hydrogen fuel. This aspect of the flame cells is reflected in the joint probability distribution of temperature and hydrogen shown Fig. 4. Of course, most of the gas in the experiment is either fresh or burnt, so the greatest probability density lies at the upper left (fresh) and lower right (burnt) corners

of this figure. At the burnt corner, note, the bulk of the distribution lies slightly above the horizontal axis indicating that not all the fuel is consumed. The exiting gas contains approximately $1.7 \%$ hydrogen by volume.

For each temperature between that of the fresh and burnt gases, Fig. 4 shows a range of hydrogen mole fractions in the gas that is passing through the flame. Consistent with the thermo-diffusive instability, Fig. 4 shows that the distribution of fuel in the flame zone approaches that of a richer mixture, in this case of an adiabatic, flat flame with $\phi=0.28$. The reactions in the flame cell therefore may approximate those of a richer, flat flame.

Figure 5 (left) shows the integral reaction network for hydrogen in the flame cell, and shows (right) the integrated reaction rates of progress for both the cell and the flat flame. The two flames share nine of their ten strongest reactions, although the individual ranking by integrated rates of progress is somewhat different. The most notable change in the flame cell from the richer, flat flame is the dominance of the backward direction of reaction 86, $2 \mathrm{OH} \rightleftharpoons \mathrm{O}+\mathrm{H}_{2} \mathrm{O}$. This reaction actually consumes a good amount of the final overall product. Examination of the spatial distribution of the rate of progress (not shown) reveals that reaction 86 is active in the flame zone, that is, in the region of high fuel consumption around the flame cell shown in Fig. 2.

\section{Conclusions}

This paper has shown that other forms of freely propagating, laminar flames can be steady and stable besides flat flames and flame balls. Several differences are apparent between these flame cells and the other two kinds of canonical, laminar flames. 
- Direct numerical simulations of premixed, ultra-lean, hydrogen-air flames in domains with periodic boundaries reveal the flames naturally evolve to flame cells. Thus the resulting flame cells are stable to perturbations in two spatial dimensions, whereas flame balls and flat flames are stable only to one-dimensional perturbations.

- For the same fuel equivalence ratio, single flame cells are able to adapt to a range of domain widths, the widest having almost double the size of the narrowest.

- Both the flame balls and the flame cells are robust in ultra-lean mixtures, that is, in mixtures that would not support a flat flame with radiative losses.

- The flame cells are stabilized by heat loss through convection, whereas flame balls, are stabilized by heat loss through radiation.

- The flame cells propagate at a speed that is an order of magnitude faster than has been observed of flame balls, $\mathcal{O}(1) \mathrm{cm} / \mathrm{sec}$ versus $\mathcal{O}(1) \mathrm{cm} / \mathrm{min}$.

- There is an inverse relationship between cell size and cell speed, with the smaller cells propagating slightly faster than the larger cells.

Since the flame cells are stable to higher dimensional perturbations than flame balls or flat flames, it is reasonable to infer that real, three-dimensional premixed flames have a natural tendency to exhibit the flame cells. Thus, flame cells may be a more promising as a theoretical basis from which to develop models of premixed combustion than the one-dimensional flame balls and flat flames, particularly under the ultra-lean conditions considered here. The lack of models for ultra-lean hydrogen combustion has been identified as a major deficiency of engineering simulations for nuclear reactor fire safety [48]. 


\section{Acknowledgments}

This work was supported by the Director, Office of Science, Office of Advanced Scientific Computing, of the U.S. Department of Energy under Contract No. DE-AC02-05CH11231. The author wishes to thank F. A. Williams for helpful discussions.

\section{References}

[1] L. Kagan, S. Minaev, G. Sivashinsky, Math. Comput. Simulation 65 (2004) 511-520.

[2] M.-S. Wu, P. D. Ronney, R. O. Colantonio, D. M. VanZandt, Combust. Flame 116 (1999) 387-397.

[3] G. H. Markstein (Ed.), Nonsteady Flame Propagation, Pergamon Press, Oxford, 1964.

[4] B. Lewis, G. von Elbe, Combustion, Flames and Explosions of Gases, Academic Press, New York, 1961.

[5] M. Hertzberg, Prog. Energy Combust. Sci. 15 (1989) 203-239.

[6] J. Buckmaster, M. Smooke, V. Giovangigli, Combust. Flame 94 (1993) 113-124.

[7] J. Buckmaster, G. Joulin, P. Ronney, Combust. Flame 79 (1990) 381-392.

[8] J. Buckmaster, G. Joulin, P. Ronney, Combust. Flame 84 (1991) 411-422.

[9] I. Glassman, Combustion, 3rd Edition, Academic Press, 1996.

[10] E. von Lavante, R. A. Strehlow, Combust. Flame 49 (1983) 123-140.

[11] B. Bregeon, A. S. Gordon, F. A. Williams, Combust. Flame 33 (1978) 33-45.

[12] T. Mitani, F. A. Williams, Combust. Flame 39 (1980) 169-190.

[13] J. Buckmaster, P. Ronney, Proc. Combust. Inst. 27 (1998) 2603-2610. 
[14] M. S. Day, J. B. Bell, Combust. Theory Modelling 4 (2000) 535-556.

[15] R. G. Rehm, H. R. Baum, N. B. S. J. Res. 83 (1978) 297-308.

[16] A. Majda, J. A. Sethian, Combust. Sci. Technol. 42 (1985) 185-205.

[17] M. J. Berger, P. Colella, J. Comput. Phys. 82 (1) (1989) 64-84.

[18] A. S. Almgren, J. B. Bell, P. Colella, L. H. Howell, M. L. Welcome, J. Comput. Phys. 142 (1998) 1-46.

[19] R. B. Pember, L. H. Howell, J. B. Bell, P. Colella, W. Y. Crutchfield, W. A. Fiveland, J. P. Jessee, Combust. Sci. Technol. 140 (1998) 123-168.

[20] J. B. Bell, M. S. Day, I. G. Shepherd, M. Johnson, R. K. Cheng, J. F. Grcar, V. E. Beckner, M. J. Lijewski, Proc. Natl. Acad. Sci. USA 102 (29) (2005) 10006-10011.

[21] J. B. Bell, M. S. Day, J. F. Grcar, M. J. Lijewski, J. F. Driscoll, S. F. Filatyev, Proc. Combust. Inst. 31 (2007) 1299-1307.

[22] A. Ern, V. Giovangigli, Multicomponent Transport Algorithms, Vol. m24 of Lecture Notes in Physics, SpringerVerlag, Berlin, 1994.

[23] A. Ern, V. Giovangigli, Phys. rev. E 53 (1996) 485-492.

[24] C. T. Bowman et al., GRI-Mech 2.11, available at http://www.me.berkeley.edu/gri_mech.

[25] M. Abid, M. S. Wu, J. B. Liu, P. D. Ronney, M. Ueki, K. Maruta, H. Kobayashi, T. Niioka, D. M. VanZandt, Combust. Flame 116 (1999) 348-359.

[26] R. J. Kee, J. F. Grcar, M. D. Smooke, J. A. Miller, PREMIX: A Fortran Program for Modeling Steady, Laminar, One-Dimensional Premixed Flames, Technical Report SAND85-8240, Sandia National Laboratories, Livermore (1983). 
[27] J. B. Bell, M. S. Day, J. F. Grcar, M. J. Lijewski, Comm. App. Math. Comput. Sci. 1 (1) (2005) 29-52.

[28] G. Patnaik, K. Kailasanath, K. J. Laskey, E. S. Oran, Proc. Combust. Inst. 22 (1989) 1517-1526.

[29] G. Patnaik, K. Kailasanath, The Stability and Structure of Lean Hydrogen-Air Flames: Effects of Gravity, Memorandum Report 6649, Naval Research Laboratory, Washington (1990).

[30] M. Tanahashi, M. Fujimura, T. Miyauchi, Proc. Combust. Inst. 28 (2000) 529-535.

[31] J. B. Bell, R. K. Cheng, M. S. Day, I. G. Shepherd, Proc. Combust. Inst. 31 (2007) 1309-1317.

[32] J. F. Grcar, J. B. Bell, M. S. Day, Proc. Combust. Inst. 32, accepted for publication.

[33] D. M. Michelson, G. I. Sivashinsky, Acta Astronautica 4 (1977) 1207-1221.

[34] B. Denet, P. Haldenwang, Combust. Sci. Technol. 86 (1992) 199-221.

[35] B. Denet, P. Haldenwang, Combust. Sci. Technol. 104 (1995) 143-167.

[36] S. Kadowaki, T. Hasegawa, Prog. Energy Combust. Sci. 31 (2005) 193-241.

[37] S. Kadowaki, Combust. Flame 143 (2005) 174-182.

[38] J. Yuan, Y. Ju, C. K. Law, Phys. Fluids 17 (7) (2005) 074106.

[39] W. Gerlinger, K. Schneider, J. Föhlich, H. Bockhorn, Combust. Flame 132 (2003) 247-271.

[40] L. Kagan, G. Sivashinsky, Combust. Flame 108 (1997) 220-226.

[41] V. Giovangigli, M. D. Smooke, Combust. Sci. Technol. 87 (1992) 241-256.

[42] D. Lozinski, J. Buckmaster, R. P., Combust. Flame 97 (1994) 301-316.

[43] J. Buckmaster, S. Weeratunga, Combust. Sci. Technol. 35 (1983) 287-296.

[44] B. Deshaires, G. Joulin, Combust. Sci. Technol. 37 (1984) 99-116. 
[45] I. Brailovsky, G. Sivashinsky, Combust. Flame 110 (1997) 524-529.

[46] F. A. Williams, J. F. Grcar, Proc. Combust. Inst. 32, accepted for oral presentation.

[47] D. Vaynblat, M. Matalon, SIAM J. Appl. Math. 60 (2) (2000) 679-702.

[48] W. Breitung et al., Nuclear Engineering and Design 253 (2005) 253-270. 
Table 1: Outcomes of the experiments.

\begin{tabular}{|c|c|}
\hline $\begin{array}{c}\text { domain } \\
\text { width }\end{array}$ & $\begin{array}{c}\text { state after 10 } \\
\text { s model time }\end{array}$ \\
\hline \hline $2.0 \mathrm{~cm}$ & unphysical flat flame \\
2.5 & unphysical flat flame \\
\hline 3.0 & steady flame cell \\
3.5 & steady flame cell \\
4.0 & steady flame cell \\
4.5 & steady flame cell \\
\hline 5.0 & laterally precessing \\
5.5 & laterally precessing \\
\hline 6.0 & oscillating \\
\hline 7.5 & two cells \\
\hline
\end{tabular}




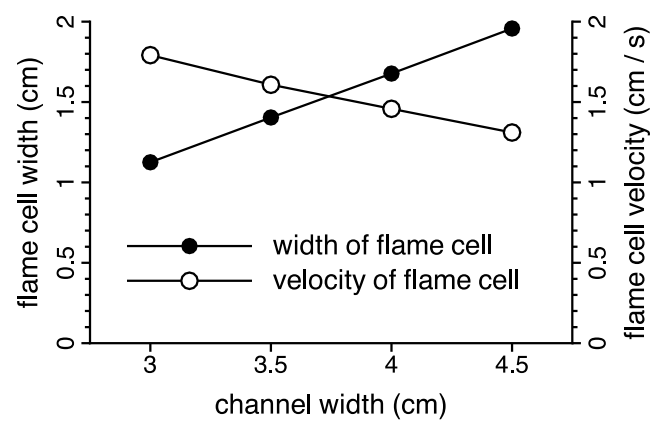

Figure 1: Sizes and velocities of the steady flame cells.

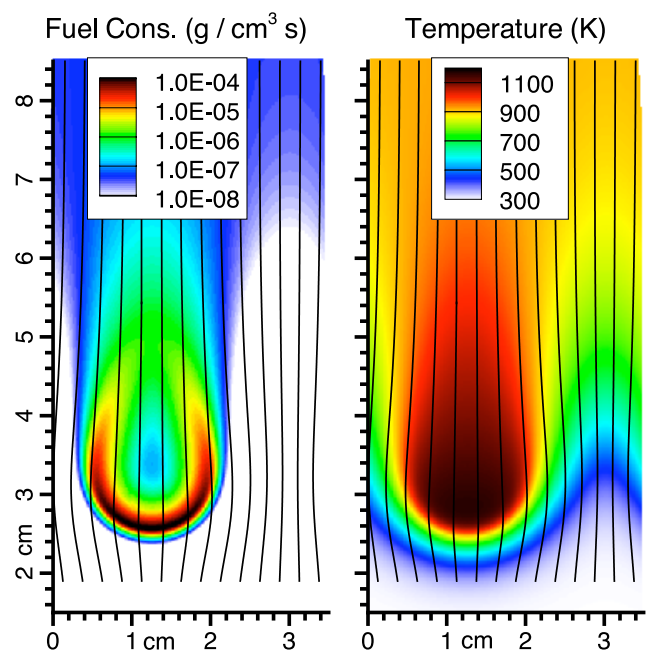

Figure 2: Fuel consumption and temperature throughout the flame cell in the domain of width $3.5 \mathrm{~cm}$. Fluid streamlines are superimposed on each figure.

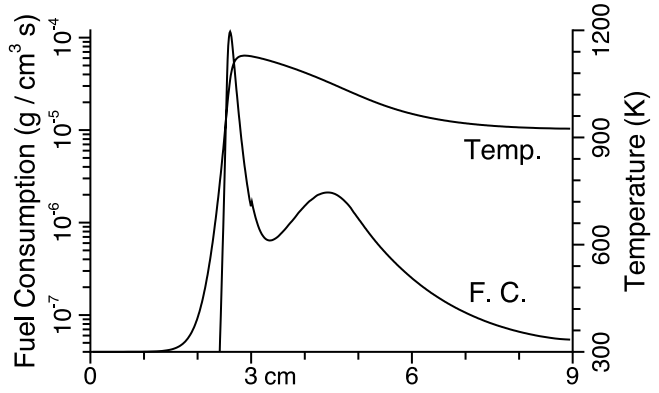

Figure 3: Fuel consumption and temperature on the centerline of the flame cell in the domain of width $3.5 \mathrm{~cm}$. 


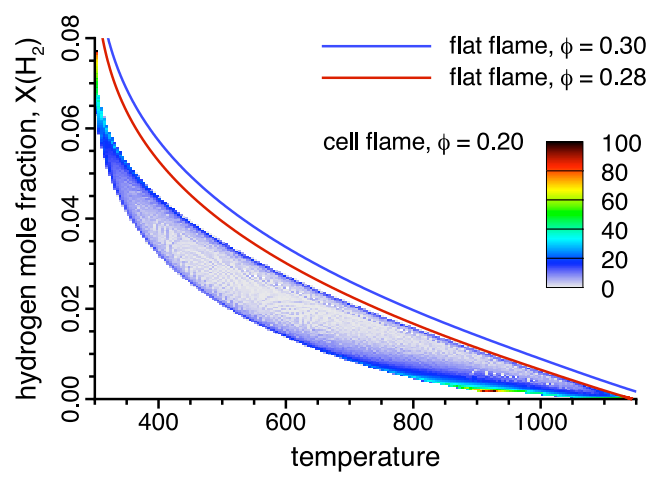

Figure 4: Joint probability density of temperature and fuel mole fraction in the flame cell for the domain of width $3.5 \mathrm{~cm}$. Superimposed on the distribution is the temperature-fuel curve for two adiabatic, flat flames. Density is depicted in terms of percentiles of the distribution.

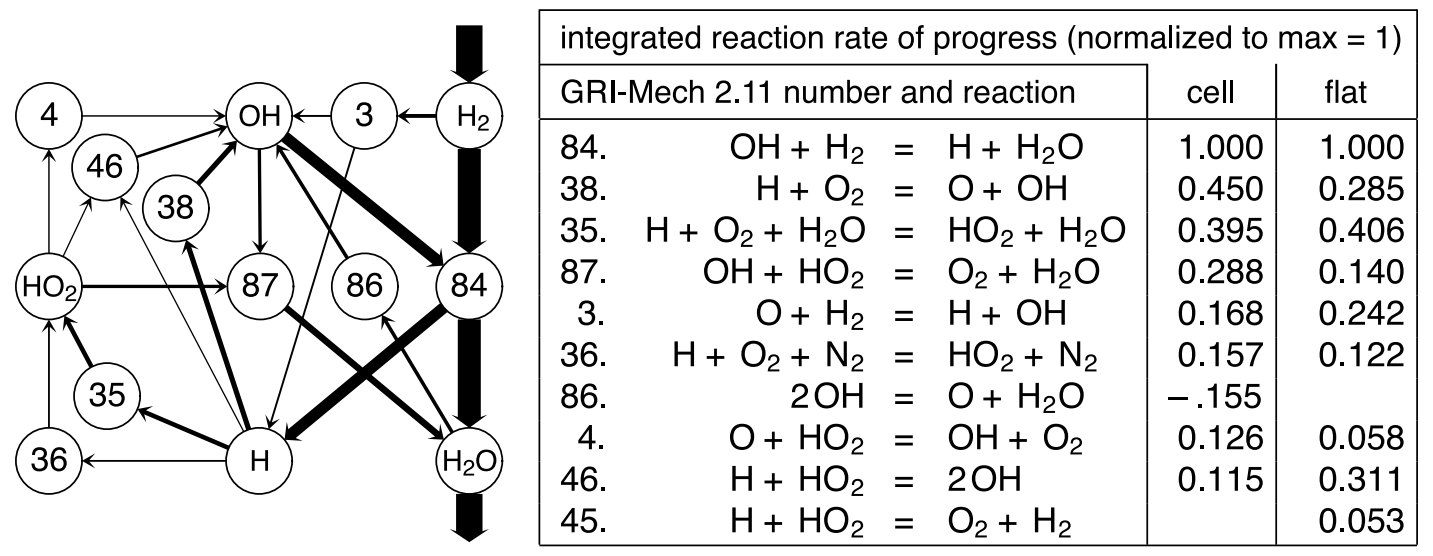

Figure 5: (left) Integrated reaction network for hydrogen in the flame cell for the domain of width $3.5 \mathrm{~cm}$ and $\phi=0.2$. The numbers in the network refer to the GRI-Mech 2.11 reactions moving hydrogen atoms among the species. The thickness of an arrow corresponds to the moles of hydrogen atoms exchanged. All arrows are shown that are at least $2 \%$ of the thickest. (right) Integrated reaction rates of progress for the flame cell of $\phi=0.2$, and for the adiabatic flat flame of $\phi=0.28$. All reactions are shown whose integrals are at least $3 \%$ of the largest. 


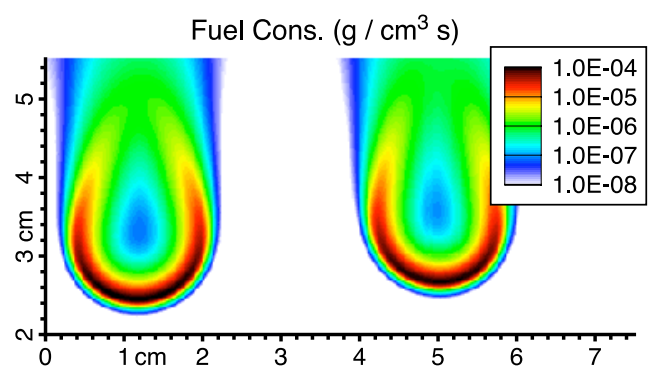

Figure 6: Fuel consumption in a pair of adjacent flame cells that persist for $4.5 \mathrm{~s}$ in the domain of width $7.5 \mathrm{~cm}$. 